\title{
Lessons of the month 1: Cardiac tamponade: don't forget the pancreas
}

\author{
Authors: Mallikarjun Patil, ${ }^{A}$ Syed Shafiq, ${ }^{B}$ Sunu S Kurien ${ }^{B}$ and Harshad Devarbhavi ${ }^{C}$
}

Cardiac tamponade is a medical emergency, caused by rapid accumulation of fluid in the pericardium resulting in reduced ventricular filling which may result in pulmonary oedema, shock and death. The common causes of cardiac tamponade include malignancy, uraemia, infectious/idiopathic pericarditis, connective tissue diseases, post-cardiac surgery etc. Early recognition and treatment of the underlying cause of the tamponade along with pericardiocentesis improves the prognosis, otherwise untreated cardiac tamponade universally results in death. We report a rare case of 32-year-old man, who presented with cardiac tamponade due to a pancreaticopericardial fistula secondary to pancreatitis and was successfully treated by endoscopic therapy.

KEYWORDS: cardiac tamponade, pancreatitis, pancreatic fistula, endoscopic retrograde pancreatography

DOI: 10.7861/clinmed.2021-0078

\section{Case presentation}

A 32-year-old man presented to the emergency department with progressively increasing dyspnoea of 1-week duration. He was well until a week previously, then developed exertional dyspnoea associated with retrosternal pain, which progressed to dyspnoea at rest. He denied history of fever, cough, night sweats or weight loss. He gave no history of any significant illness in the past or any illicit drug use. He had chronic alcoholism and did not smoke. On clinical examination he was afebrile and dyspnoeic at rest with oxygen saturation of $95 \%$ on $5 \mathrm{~L}$ nasal oxygen. Examination showed he had tachycardia (pulse of 110 beats per minute), hypotension (blood pressure of $90 / 50 \mathrm{mmHg}$ ), elevated jugular venous pressure $(9 \mathrm{~cm})$ and pulsus paradoxus of $15 \mathrm{mmHg}$. Cardiac examination revealed muffled heart sounds and respiratory examination demonstrated decreased breath sounds at both lung bases.

Authors: ${ }^{\text {A }}$ rofessor, St John's Medical College Hospital, Bangalore, India; ' assistant professor, St John's Medical College Hospital, Bangalore, India; ' professor and head of the Department of Gastroenterology, St John's Medical College Hospital, Bangalore, India
His clinical features and physical examination findings were of concern of pericardial effusion with cardiac tamponade. Bedside electrocardiography (ECG) revealed tachycardia with low voltage complexes. Chest $\mathrm{X}$-ray and contrast enhanced computed tomography of the chest showed large pericardial effusion and bilateral minimal pleural effusion (Fig 1a and b). Bedside echocardiography showed pericardial effusion with physiologic features of tamponade. In view of pericardial effusion with tamponade causing haemodynamic compromise, urgent pericardiocentesis was done under echocardiographic guidance and approximately 1 litre of brownish fluid removed. After pericardiocentesis, the patient's haemodynamic parameters improved. Pericardial fluid analysis was suggestive of an exudative pattern, with 710 white blood cells/ $\mu$ L. Fluid adenosine deaminase (ADA) level was $8 \mathrm{U} / \mathrm{L}$, and cytology examination for malignant cells was negative. The next day, there was a rapid re-accumulation of pericardial fluid; one litre of pericardial fluid was removed with the help of a pericardial catheter which was subsequently kept in situ. His laboratory parameters such as complete blood counts, liver function test, renal function tests (blood urea and creatinine) were normal. Autoimmune serology
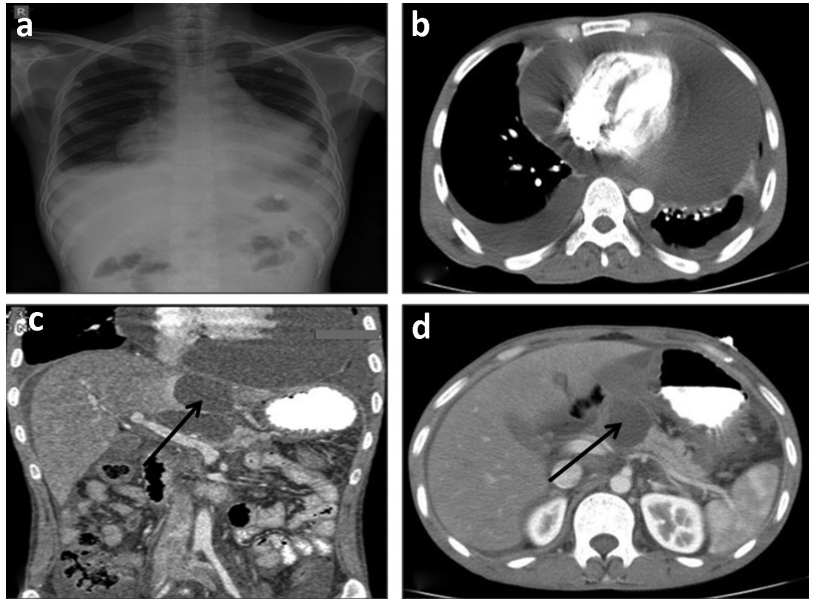

Fig 1. a) Chest X-ray showing cardiomegaly. b) Computed tomography of the chest showing large pericardial effusion and minimal pleural effusion. $c$ and d) Computed tomography of the abdomen showing pancreatitis with peripancreatic fluid collection and extension of fluid collection up to the diaphragm. 

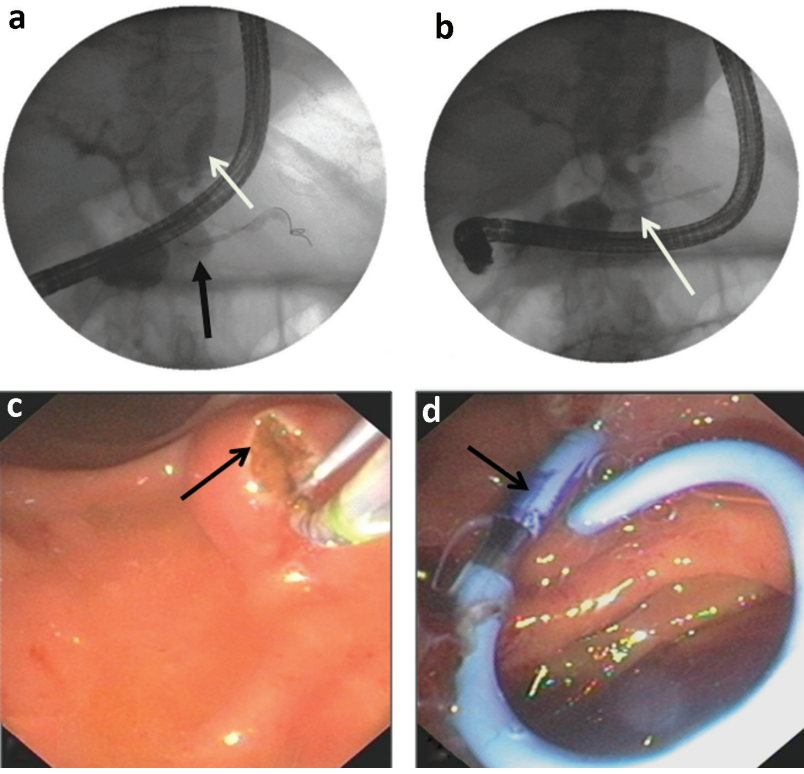

Fig 2. a) Pancreaticography showing contrast leak from the pancreatic duct and leak traversing towards the diaphragm and pericardium. b) Fluoroscopy showing pancreatic stent placed across the leak. $c$ and d) Endoscopic image showing pancreatic sphincterotomy and pancreatic stent.

like antinuclear antibody and rheumatoid factor were negative. His HIV serology was negative. On the third day of admission, he started complaining of severe epigastric pain radiating to the back. His serum amylase and lipase were raised (1,021 U/L and 2,064 U/L, respectively). Contrast enhanced computed tomography of the abdomen revealed pancreatitis with peripancreatic fluid collection traversing to under the diaphragm (Fig 1c and d). Subsequently, pericardial fluid amylase estimation showed a level of 51,988 U/L. A diagnosis of pancreatitis with a possible pancreatico-pericardial fistula causing cardiac tamponade was established.

The patient was treated with intravenous fluids and analgesics. Since pericardial fluid drainage was persistently about $1 \mathrm{~L}$ per day, an endoscopic retrograde pancreatography (ERP) was planned together with placement of a pancreatic stent across the leak. ERP demonstrated a pancreatic ductal leak from the body region with contrast traversing upwards towards the diaphragm, findings suggestive of a pancreatico-pericardial fistula (Fig 2a). Pancreatic sphincterotomy was carried out followed by a placement of a pancreatic stent (7 French; $7 \mathrm{~cm}$ single pigtail) bridging the ductal disruption (Fig 2b, c and d). Pericardial fluid drainage gradually decreased over 3 days and the pericardial catheter was removed on day 5 following ERP. The patient's abdominal pain also improved simultaneously. Follow up chest X-ray (Fig 3a and b) and echocardiography after 6 weeks showed complete resolution of pericardial effusion. The pancreatic stent was removed after 8 weeks. The patient was continuing to do well after 1 year of follow-up.

\section{Discussion}

Cardiac tamponade occurs due to accumulation of pericardial fluid under pressure which impairs cardiac filling leading to
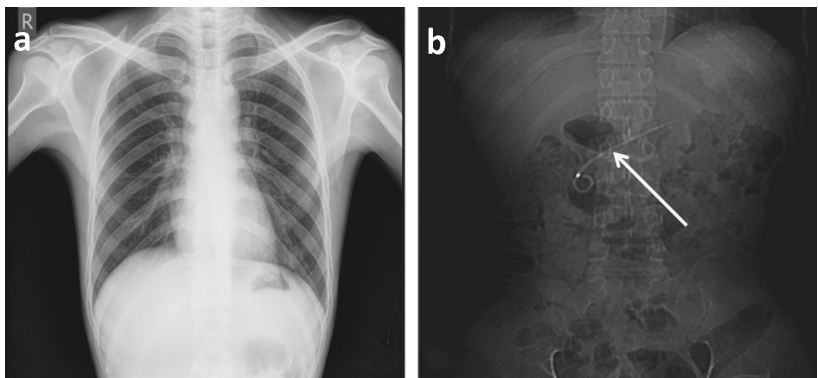

Fig 3. a) Chest X-ray showing normal cardiac silhouette. b) Abdominal $X$-ray showing pancreatic stent.

haemodynamic compromise. The various causes of tamponade are pericarditis secondary to infections (bacterial, fungal and viral), neoplastic, post-cardiac surgery, renal failure, autoimmune and collagen vascular diseases (systemic lupus erythematosus and rheumatoid arthritis), idiopathic pericarditis and aortic dissection. However, cardiac tamponade secondary to a pancreaticopericardial fistula is rare and very few cases have been reported.

${ }^{1-3}$ To the best of our knowledge, this is the fourth case of cardiac tamponade due to pancreatic pericardial effusion which was managed successfully by endoscopic therapy. ${ }^{4}$ Acute and chronic pancreatitis can lead to pancreatic ductal disruption causing an internal fistula, which is an abnormal communication between the pancreatic duct and viscera or pleural or peritoneal cavity. Anterior pancreatic ductal disruption leads to pancreatic ascites and posterior disruption to pleural effusion or pericardial effusion. Pancreatic internal fistulae are seen in $0.4 \%$ cases of pancreatitis. ${ }^{5}$ The pathogenesis of pericardial effusion in pancreatitis is not clear and has been ascribed to chemical pericarditis from circulating pancreatic proteolytic enzymes or due to ductal disruption/ penetration of the pseudocyst of pancreas into the pericardial cavity. Asymptomatic, self-limiting mild pericardial effusion is seen in $18 \%$ to $48 \%$ of patients with acute pancreatitis due to alcohol, which subsides with improvement of pancreatitis. ${ }^{6}$ Pericardial effusion caused by a pancreatic fistula is rare, and are usually large, and symptomatic and may require interventions either endoscopic therapy or surgery. There is no standard guideline, however, given the rarity of this complication. In the published literature, pancreatic pericardial effusion was commonly seen in males. Two-thirds were people with alcoholism and a past history of pancreatitis.

In earlier periods, pancreatic fistulae were treated conservatively by keeping the patient nil by mouth, administration of total parenteral nutrition and octreotide therapy to decrease pancreatic secretion. However, response to conservative treatment was $25 \%-60 \%$ and this was associated with a mortality rate of $1 \%-25 \%{ }^{7}$. Furthermore, such measures required prolonged hospitalisation. With the advancement in the endoscopic treatment in various pancreatic diseases, pancreatic fistulae are now a day's treatment by restoration of anatomical continuity by pancreatic stent placement with success rate of $50 \%-90 \%$ requiring only very few days of hospitalisation. Failure to respond to endoscopic therapy requires surgery. ${ }^{8}$

\section{Conclusion}

Pericardial effusion / cardiac tamponade due to pancreatitis is very rare. The diagnosis needs to be suspected in people with 
alcoholism with unexplained pericardial effusion and in patients with pancreatitis who develop dyspnoea and shock. Diagnosis is established by measuring amylase levels in the pericardial fluid and cross-sectional abdominal imaging that demonstrates features of pancreatitis with associated pancreatic pseudocyst or peripancreatic fluid collection. First-line therapy for persistent, symptomatic pericardial effusion should be endoscopic therapy.

\section{References}

1 Bhatt VR, Koirala A, Wetz RV et al. Cardiac tamponade in acute pancreatitis. BMJ Case Rep 2011;2011:bcr0820103287.

2 Lamparter S, Sundermann H. Swinging heart in acute pancreatitis. Am J Med Sci 2013;346:160-1.

3 Gupta A, Awerbuch E. Massive loculated pericardial effusion with tamponade physiology associated with acute pancreatitis. Chest 2016;150:235A.

4 Khan MS, Shahbaz N, Zia HA et al. Pancreaticopericardial fistula: a case report and literature review. Case Rep Crit Care 2016;2016: 7169341.
5 Bhasin DK, Rana SS, Siyad I, Poddar U et al. Endoscopic transpapillarynasopancreatic drainage alone to treat pancreatic ascites and pleural effusion. J Gastroenterol Hepatol 2006;21:1059-64.

6 Maringhini A, Ciambra M, Patil R et al. Ascites, pleural, and pericardial effusions in acute pancreatitis. A prospective study of incidence, natural history, and prognostic role. Dig Dis Sci 1996;41: 848-52.

7 Parekh D, Segal I. Pancreatic ascites and effusion. Risk factors for failure of conservative therapy and the role of octreotide. Arch Surg 1992;127:707-12.

8 Eckhauser F, Raper SE, Knol JA, Muiholland MW. Surgical management of pancreatic pseudocysts, pancreatic ascites, and pancreaticopleural fistulae. Pancreas 1991;6:S66-75.

Address for correspondence: Prof Mallikarjun Patil, Department of Gastroenterology and Hepatology, St John's Medical College, Sarjapur Road, Bangalore 560034, Karnataka, India. Email:drmalli_arjun@yahoo.co.in 